\title{
A New Entry of Nucleophiles in Rhodium-Catalyzed Asymmetric 1,4-Addition Reactions: Addition of Organozinc Reagents for the Synthesis of 2-Aryl-4- Piperidones
}

\author{
Ryo Shintani, Norihito Tokunaga, Hidenori Doi, and Tamio Hayashi* \\ Department of Chemistry, Graduate School of Science, Kyoto University, \\ Sakyo, Kyoto 606-8502, Japan
}

\section{Supporting Information}

\section{General}

All air- and moisture-sensitive manipulations were carried out with standard Schlenk techniques under nitrogen or in a glove box under argon.

THF, 1,4-dioxane, and toluene were distilled over benzophenone ketyl under nitrogen.

4-piperidone monohydrate hydrochloride (TCI), phenylboronic acid (TCI), benzyl chloroformate (Wako Chemicals), Pd/C (Wako Chemicals; $10 \mathrm{wt} \%$ ), $t$-BuLi (Kanto Chemicals; $1.48 \mathrm{M}$ solution in pentane), allyl bromide (TCI), 2-cyclohexen-1one (Wako Chemicals), and 5-methyl-3-hexen-2-one (TCI) were used as received. Pivaloyl chloride (TCI) was distilled under vacuum prior to use. Ph-9-BBN, $\mathrm{PhTi}(\mathrm{O} i-\mathrm{Pr})_{3},{ }^{2} \mathrm{Rh}(\mathrm{acac})\left(\mathrm{C}_{2} \mathrm{H}_{4}\right)_{2},{ }^{3} \quad[\mathrm{Rh}(\mathrm{OMe})(\mathrm{cod})]_{2},{ }^{4}$ and $[\mathrm{RhCl}((\mathrm{R}) \text {-binap })]_{2}{ }^{5}$ were synthesized following the literature procedure.

All other chemicals and solvents were purchased from Aldrich, Wako Chemicals, TCI, or Kanto Chemicals and used as received.

\section{Synthesis of Substrates}

The yields have not been optimized.

Benzyl 3,4-dihydro-4-oxo-1(2H)-pyridinecarboxylate (1) (CAS 185847-84-1)<smiles>O=C1C=CN(C(=O)OCc2ccccc2)CC1</smiles>

\footnotetext{
${ }^{1}$ Kramer, G. W.; Brown, H. C. J. Organomet. Chem. 1974, 73, 1.

${ }^{2}$ Weidmann, B.; Widler, L.; Olivero, A. G.; Maycock, C. D.; Seebach, D. Helv. Chim. Acta 1981, 64, 357.

${ }^{3}$ Cramer, R. Inorg. Synth. 1974, 15, 16.

${ }^{4}$ Uson, R.; Oro, L. A.; Cabeza, J. A. Inorg. Synth. 1985, 23, 126.

${ }^{5}$ Hayashi, T.; Takahashi, M.; Takaya, Y.; Ogasawara, M. J. Am. Chem. Soc. 2002, 124, 5052 .
} 
This was synthesized from 4-piperidone monohydrate hydrochloride and benzyl chloroformate, following the literature procedure for ethyl 3,4-dihydro-4-oxo-1 $(2 \mathrm{H})$ pyridinecarboxylate. ${ }^{6}$ White solid, $40 \%$ overall yield.

${ }^{1} \mathrm{H}$ NMR $\left(\mathrm{CDCl}_{3}\right): \delta 7.85$ (bs, 1H), 7.40-7.37 (m, 5H), 5.35 (bs, $\left.1 \mathrm{H}\right), 5.27(\mathrm{~s}, 2 \mathrm{H}), 4.05$ $\left(\mathrm{t},{ }^{3} J_{\mathrm{HH}}=7.3 \mathrm{~Hz}, 2 \mathrm{H}\right), 2.56\left(\mathrm{t},{ }^{3} \mathrm{JHH}_{\mathrm{HH}}=7.3 \mathrm{~Hz}, 2 \mathrm{H}\right) .{ }^{13} \mathrm{C} \mathrm{NMR}\left(\mathrm{CDCl}_{3}\right): \delta 193.2,152.5$, $143.3,134.8,128.74,128.68,128.4,107.7,69.0,42.5,35.6$.

\section{General Procedure for the Preparation of Arylzinc Chlorides}

$t$-BuLi (13.5 mL, $20.0 \mathrm{mmol} ; 1.48 \mathrm{M}$ solution in pentane) was added dropwisely to a solution of aryl bromide $(10.0 \mathrm{mmol})$ in THF $(5.0 \mathrm{~mL})$ at $-78{ }^{\circ} \mathrm{C}$. The resulting mixture was stirred for $30 \mathrm{~min}$ at $-78{ }^{\circ} \mathrm{C}$, and then $15 \mathrm{~min}$ at room temperature. The aryllithium solution thus obtained was added to a suspension of $\mathrm{ZnCl}_{2}(1.45 \mathrm{~g}, 10.6$ $\mathrm{mmol})$ in THF $(2.5 \mathrm{~mL})$ at $0{ }^{\circ} \mathrm{C}$, with the aid of additional THF $(5.0 \mathrm{~mL})$. The reaction mixture was stirred for $15 \mathrm{~min}$ at room temperature, and was then concentrated under vacuum to remove pentane. THF $(5.0 \mathrm{~mL})$ was added to the resulting mixture to obtain a solution of arylzinc chloride in THF. The concentration was determined by acid-base titration with methyl orange (typically around $0.5 \mathrm{M}$ ).

\section{1,4-Addition Reactions}

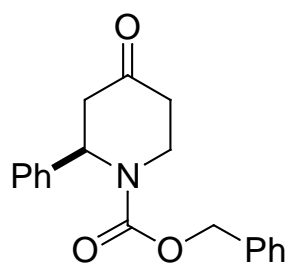

Procedure for Table 1, Entry 1. (CAS 335266-05-2)

A solution of $1(57.8 \mathrm{mg}, 0.25 \mathrm{mmol}), \mathrm{PhB}(\mathrm{OH})_{2}(91.4 \mathrm{mg}, 0.75 \mathrm{mmol})$, $\mathrm{Rh}$ (acac) $\left(\mathrm{C}_{2} \mathrm{H}_{4}\right)_{2}(1.9 \mathrm{mg}, 7.3 \mu \mathrm{mol})$, and $(R)$-binap $(5.1 \mathrm{mg}, 8.2 \mu \mathrm{mol})$ in 1,4-dioxane $(1.0 \mathrm{~mL})$ and $\mathrm{H}_{2} \mathrm{O}(0.1 \mathrm{~mL})$ was stirred for $2 \mathrm{~h}$ at $100{ }^{\circ} \mathrm{C}$. The reaction mixture was directly passed through a pad of silica gel with $\mathrm{Et}_{2} \mathrm{O}$ and the solvent was removed under vacuum. The residue was purified by silica gel PTLC with $\mathrm{Et}_{2} \mathrm{O} /$ hexane $=$ $2 / 1$ to afford the desired 1,4-adduct (R)-2a as a white solid (25.6 mg, $83 \mu \mathrm{mol} ; 33 \%$ yield).

The ee was determined on a Daicel Chiralcel OD-H column with hexane : isopropanol $=90: 10$, flow $=1.0 \mathrm{~mL} / \mathrm{min}$. Retention times: $20.9 \mathrm{~min}[(S)$ enantiomer], $27.0 \mathrm{~min}[(R)$-enantiomer]. 96\% ee.

${ }^{1} \mathrm{H}$ NMR $\left(\mathrm{CDCl}_{3}\right): \delta$ 7.36-7.31 (m, 7H), 7.29-7.24 (m, 3H), $5.84(\mathrm{bs}, 1 \mathrm{H}), 5.25\left(\mathrm{~d},{ }^{2} J_{\mathrm{HH}}\right.$ $=12.3 \mathrm{~Hz}, 1 \mathrm{H}), 5.20\left(\mathrm{~d},{ }^{2} J_{\mathrm{HH}}=12.3 \mathrm{~Hz}, 1 \mathrm{H}\right), 4.28(\mathrm{bs}, 1 \mathrm{H}), 3.19\left(\mathrm{t},{ }^{3} J_{\mathrm{HH}}=11.0 \mathrm{~Hz}, 1 \mathrm{H}\right)$, $2.99\left(\mathrm{~d},{ }^{2} J_{\mathrm{HH}}=13.8 \mathrm{~Hz}, 1 \mathrm{H}\right), 2.86\left(\mathrm{dd},{ }^{2} J_{\mathrm{HH}}=15.5 \mathrm{~Hz}\right.$ and $\left.{ }^{3} J_{\mathrm{HH}}=7.0 \mathrm{~Hz}, 1 \mathrm{H}\right), 2.57-2.50$ $(\mathrm{m}, 1 \mathrm{H}), 2.37\left(\mathrm{~d},{ }^{2} J_{\mathrm{HH}}=15.8 \mathrm{~Hz}, 1 \mathrm{H}\right) .{ }^{13} \mathrm{C}$ NMR $\left(\mathrm{CDCl}_{3}\right): \delta 207.0,155.3,139.6,136.2$, $128.7,128.5,128.1,127.9,127.6,126.6,67.7,54.5,44.1,40.4,38.8$.

\footnotetext{
${ }^{6}$ Kozikowski, A. P.; Park, P.-u. J. Org. Chem. 1990, 55, 4668.
} 


\section{Procedure for Table 1, Entry 2.}

A solution of $1(46.4 \mathrm{mg}, 0.20 \mathrm{mmol}), \mathrm{PhB}(\mathrm{OH})_{2}(73.2 \mathrm{mg}, 0.60 \mathrm{mmol})$, and $[\mathrm{Rh}(\mathrm{OH})((R) \text {-binap })]_{2}(4.5 \mathrm{mg}, 6.0 \mu \mathrm{mol} \mathrm{Rh})$ in 1,4-dioxane $(0.7 \mathrm{~mL})$ and $\mathrm{H}_{2} \mathrm{O}(70 \mu \mathrm{L})$ was stirred for $5 \mathrm{~h}$ at $50{ }^{\circ} \mathrm{C}$. The reaction mixture was directly passed through a pad of silica gel with $\mathrm{Et}_{2} \mathrm{O}$ and the solvent was removed under vacuum. The residue was purified by silica gel PTLC with $\mathrm{Et}_{2} \mathrm{O} /$ hexane $=2 / 1$ to afford the desired 1,4-adduct $(R)-2 a$ as a white solid ( $48.6 \mathrm{mg}, 0.157 \mathrm{mmol} ; 78 \%$ yield). $98 \%$ ee

\section{Procedure for Table 1, Entry 3.}

A solution of $[\mathrm{Rh}(\mathrm{OMe})(\mathrm{cod})]_{2}(1.5 \mathrm{mg}, 6.0 \mu \mathrm{mol} \mathrm{Rh})$ and $(R)$-binap (4.1 mg, 6.6 $\mu \mathrm{mol})$ in toluene $(0.5 \mathrm{~mL})$ was stirred for $2 \mathrm{~min}$ at $80^{\circ} \mathrm{C} .1(46.3 \mathrm{mg}, 0.20 \mathrm{mmol})$ and Ph-9-BBN (44 mg, $0.22 \mathrm{mmol}$ ) were successively added to this solution, and the mixture was stirred for $1 \mathrm{~h}$ at $80{ }^{\circ} \mathrm{C}$. After quenching with $\mathrm{MeOH}(21 \mu \mathrm{L})$, the solvent was removed under vacuum, and then the residue was purified by silica gel PTLC with $\mathrm{Et}_{2} \mathrm{O} /$ hexane $=2 / 1$ to afford the desired 1,4-adduct (R)-2a as a white solid (20.5 mg, $66 \mu \mathrm{mol} ; 33 \%$ yield). $97 \%$ ee.

\section{Procedure for Table 1, Entry 4.}

A solution of 1 (46.3 mg, $0.20 \mathrm{mmol}), \operatorname{PhTi}(\mathrm{O} i-\mathrm{Pr})_{3}(97 \mathrm{mg}, 0.32 \mathrm{mmol})$, and $[\mathrm{Rh}(\mathrm{OH})((\mathrm{R}) \text {-binap })]_{2}(4.5 \mathrm{mg}, 6.0 \mu \mathrm{mol} \mathrm{Rh})$ in THF $(0.5 \mathrm{~mL})$ was stirred for $1 \mathrm{~h}$ at $20{ }^{\circ} \mathrm{C}$. After quenching with $\mathrm{MeOH}(0.1 \mathrm{~mL})$, the reaction mixture was passed through a pad of silica gel with $\mathrm{Et}_{2} \mathrm{O}$, and the solvent was then removed under vacuum (1,4-adduct $2 \mathrm{a} / 1,2$-adduct $\sim 3 / 1$ by ${ }^{1} \mathrm{H}$ NMR). The residue was purified by silica gel PTLC with $\mathrm{Et}_{2} \mathrm{O} /$ hexane $=2 / 1$ to afford the desired 1,4-adduct $(R)-2 \mathbf{a}$, which was contaminated with the undesired 1,2-adduct $(54.4 \mathrm{mg}, 0.176 \mathrm{mmol} ; 4 / 1$ mixture, $87 \%$ yield combined). 1,4-adduct: $>99.5 \%$ ee.

\section{Procedure for Table 1, Entry 5.}

$\mathrm{PhZnCl}(0.82 \mathrm{~mL}, 0.45 \mathrm{mmol} ; 0.55 \mathrm{M}$ solution in THF) was added dropwisely to a stirring solution of $1(69.4 \mathrm{mg}, 0.30 \mathrm{mmol})$ and $[\mathrm{RhCl}((R) \text {-binap })]_{2}(6.8 \mathrm{mg}, 9.0 \mu \mathrm{mol}$ $\mathrm{Rh})$ in THF $(1.0 \mathrm{~mL})$, and the mixture was stirred for $2 \mathrm{~h}$ at $20^{\circ} \mathrm{C}$. After quenching with $\mathrm{H}_{2} \mathrm{O}(0.1 \mathrm{~mL})$, the reaction mixture was passed through a pad of silica gel with $\mathrm{Et}_{2} \mathrm{O}$, and the solvent was then removed under vacuum. The residue was chromatographed on silica gel with $\mathrm{Et}_{2} \mathrm{O} /$ hexane $=1 / 1$ to afford the desired 1,4adduct $(R)-2 \mathrm{a}$ as a white solid $(88.6 \mathrm{mg}, 0.29 \mathrm{mmol} ; 95 \%$ yield $) .>99.5 \%$ ee. $[\alpha]^{20}{ }_{\mathrm{D}}$ $+74.3\left(c 1.00, \mathrm{CHCl}_{3}\right)$. The absolute configuration was assigned by analogy with equation 2.

\section{General procedure for Table 2.}

$\mathrm{ArZnCl}$ (0.30 mmol; THF solution) was added dropwisely to a stirring solution of $1(46.3 \mathrm{mg}, 0.20 \mathrm{mmol})$ and $\left[\mathrm{RhCl}((R) \text {-binap) }]_{2}(4.6 \mathrm{mg}, 6.0 \mu \mathrm{mol} \mathrm{Rh})\right.$ in THF $(0.5 \mathrm{~mL})$, and the mixture was stirred for $2 \mathrm{~h}$ at $20{ }^{\circ} \mathrm{C}$. After quenching with $\mathrm{H}_{2} \mathrm{O}(50 \mu \mathrm{L})$, the reaction mixture was passed through a pad of silica gel with $\mathrm{Et}_{2} \mathrm{O}$, and the solvent was then removed under vacuum. The residue was chromatographed on silica gel with $\mathrm{Et}_{2} \mathrm{O} /$ hexane to afford the desired 1,4-adduct 2. 
<smiles>O=C1CCN(C(=O)OCc2ccccc2)C(c2ccc(-c3ccccc3)cc2)C1</smiles>

Table 2, entry $2((\boldsymbol{R})-\mathbf{2 b})$. The reaction was stirred for $4 \mathrm{~h}$. Yellow solid. 97\% yield. The ee was determined on a Daicel Chiralpak AD column with hexane : isopropanol $=90: 10$, flow $=1.0 \mathrm{~mL} / \mathrm{min}$. Retention times: $19.6 \mathrm{~min}[(S)$ enantiomer], $24.1 \mathrm{~min}\left[(R)\right.$-enantiomer]. $>99.5 \%$ ee. $[\alpha]^{20}{ }_{\mathrm{D}}+79.6\left(c 1.32, \mathrm{CHCl}_{3}\right)$. The absolute configuration was assigned by analogy with equation 2 .

${ }^{1} \mathrm{H} \mathrm{NMR}\left(\mathrm{CDCl}_{3}\right): \delta 7.55\left(\mathrm{t},{ }^{3} \mathrm{~J}_{\mathrm{HH}}=7.1 \mathrm{~Hz}, 4 \mathrm{H}\right), 7.43\left(\mathrm{t},{ }^{3} J_{\mathrm{HH}}=7.4 \mathrm{~Hz}, 2 \mathrm{H}\right), 7.37-7.28$ $(\mathrm{m}, 8 \mathrm{H}), 5.87(\mathrm{bs}, 1 \mathrm{H}), 5.27\left(\mathrm{~d},{ }^{2} J_{\mathrm{HH}}=12.3 \mathrm{~Hz}, 1 \mathrm{H}\right), 5.21\left(\mathrm{~d},{ }^{2} J_{\mathrm{HH}}=12.3 \mathrm{~Hz}, 1 \mathrm{H}\right), 4.31$ $(\mathrm{bs}, 1 \mathrm{H}), 3.25\left(\mathrm{t},{ }^{3} J_{\mathrm{HH}}=11.3 \mathrm{~Hz}, 1 \mathrm{H}\right), 3.01\left(\mathrm{~d},{ }^{2} J_{\mathrm{HH}}=15.1 \mathrm{~Hz}, 1 \mathrm{H}\right), 2.89\left(\mathrm{dd},{ }^{2} J_{\mathrm{HH}}=15.4\right.$ $\mathrm{Hz}$ and $\left.{ }^{3} \mathrm{~J}_{\mathrm{HH}}=6.8 \mathrm{~Hz}, 1 \mathrm{H}\right), 2.58-2.52(\mathrm{~m}, 1 \mathrm{H}), 2.39\left(\mathrm{~d},{ }^{3} \mathrm{~J}_{\mathrm{HH}}=15.8 \mathrm{~Hz}, 1 \mathrm{H}\right) .{ }^{13} \mathrm{C} \mathrm{NMR}$ $\left(\mathrm{CDCl}_{3}\right): \delta 207.2,155.5,140.7,140.3,138.6,136.2,128.8,128.6,128.2,128.0,127.48$, $127.45,127.1,127.0,67.8,54.4,44.2,40.5,39.0$. Anal. Calcd for $\mathrm{C}_{25} \mathrm{H}_{23} \mathrm{NO}_{3}: \mathrm{C}, 77.90 ; \mathrm{H}$, 6.01. Found: $\mathrm{C}, 77.64 ; \mathrm{H}, 6.23$.<smiles>COc1ccc(C2CC(=O)CCN2C(=O)OCc2ccccc2)cc1</smiles>

Table 2, entry $3((R)-2 c)$. The reaction was stirred for $12 \mathrm{~h}$ with 3.0 equiv of 4 $\mathrm{MeOC}_{6} \mathrm{H}_{4} \mathrm{ZnCl}$ in the presence of $\left[\mathrm{RhCl}((\mathrm{R}) \text {-binap) }]_{2}(6 \mathrm{~mol} \% \mathrm{Rh})\right.$. Colorless oil. $90 \%$ yield. The ee was determined on a Daicel Chiralcel OD-H column with hexane : isopropanol $=90: 10$, flow $=1.0 \mathrm{~mL} / \mathrm{min}$. Retention times: $30.6 \mathrm{~min}[(S)-$ enantiomer], $36.0 \mathrm{~min}\left[(R)\right.$-enantiomer]. $99 \%$ ee. $[\alpha]_{D}^{20}+71.1$ (c 1.51, $\left.\mathrm{CHCl}_{3}\right)$. The absolute configuration was assigned by analogy with equation 2 .

${ }^{1} \mathrm{H} \mathrm{NMR}\left(\mathrm{CDCl}_{3}\right): \delta 7.39-7.32(\mathrm{~m}, 5 \mathrm{H}), 7.16\left(\mathrm{~d},{ }^{3} \mathrm{~J}_{\mathrm{HH}}=8.3 \mathrm{~Hz}, 2 \mathrm{H}\right), 6.85-6.83(\mathrm{~m}$, $2 \mathrm{H}), 5.82(\mathrm{bs}, 1 \mathrm{H}), 5.25\left(\mathrm{~d},{ }^{2} J_{\mathrm{HH}}=12.2 \mathrm{~Hz}, 1 \mathrm{H}\right), 5.20\left(\mathrm{~d},{ }^{2} J_{\mathrm{HH}}=12.2 \mathrm{~Hz}, 1 \mathrm{H}\right), 4.26(\mathrm{bs}$, $1 \mathrm{H}), 3.79(\mathrm{~s}, 3 \mathrm{H}), 3.13\left(\mathrm{t},{ }^{3} J_{\mathrm{HH}}=11.2 \mathrm{~Hz}, 1 \mathrm{H}\right), 2.95\left(\mathrm{~d},{ }^{2} J_{\mathrm{HH}}=15.4 \mathrm{~Hz}, 1 \mathrm{H}\right), 2.84(\mathrm{dd}$, ${ }^{2} J_{\mathrm{HH}}=15.5 \mathrm{~Hz}$ and $\left.{ }^{3} J_{\mathrm{HH}}=7.0 \mathrm{~Hz}, 1 \mathrm{H}\right), 2.57-2.49(\mathrm{~m}, 1 \mathrm{H}), 2.35\left(\mathrm{~d},{ }^{2} J_{\mathrm{HH}}=15.5 \mathrm{~Hz}, 1 \mathrm{H}\right)$. ${ }^{13} \mathrm{C}$ NMR $\left(\mathrm{CDCl}_{3}\right): \delta 207.4,159.0,155.4,136.3,131.5,129.6,128.6,128.2,128.0,114.1$, 67.7, 55.2, 54.1, 44.3, 40.6, 38.7. Anal. Calcd for $\mathrm{C}_{20} \mathrm{H}_{21} \mathrm{NO}_{4}: \mathrm{C}, 70.78 ; \mathrm{H}, 6.24$. Found: C, 70.56; H, 6.33 .

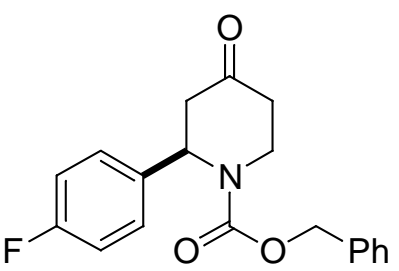

Table 2, entry 4 ((R)-2d). (CAS 414910-20-6) The reaction was stirred for $7 \mathrm{~h}$. Pale yellow oil. 91\% yield. The ee was determined on a Daicel Chiralcel OD-H column with hexane : isopropanol $=90: 10$, flow $=1.0 \mathrm{~mL} / \mathrm{min}$. Retention times: 
$26.7 \min \left[(S)\right.$-enantiomer], $36.3 \mathrm{~min}\left[(R)\right.$-enantiomer]. $>99.5 \%$ ee. $[\alpha]_{\mathrm{D}}^{20}+67.4(c 0.68$, $\left.\mathrm{CHCl}_{3}\right)$. The absolute configuration was assigned by analogy with equation 2.

${ }^{1} \mathrm{H}$ NMR $\left(\mathrm{CDCl}_{3}\right): \delta$ 7.38-7.31 (m, 5H), 7.24-7.18 (m, 2H), $7.00(\mathrm{t}, J=8.5 \mathrm{~Hz}, 2 \mathrm{H})$, $5.82(\mathrm{bs}, 1 \mathrm{H}), 5.25\left(\mathrm{~d},{ }^{2} J_{\mathrm{HH}}=12.2 \mathrm{~Hz}, 1 \mathrm{H}\right), 5.19\left(\mathrm{~d},{ }^{2} J_{\mathrm{HH}}=12.2 \mathrm{~Hz}, 1 \mathrm{H}\right), 4.28(\mathrm{bs}, 1 \mathrm{H})$, $3.16\left(\mathrm{t}^{3} \mathrm{~J}_{\mathrm{HH}}=11.1 \mathrm{~Hz}, 1 \mathrm{H}\right), 2.94\left(\mathrm{dd},{ }^{2} J_{\mathrm{HH}}=15.4 \mathrm{~Hz}\right.$ and $\left.{ }^{3} J_{\mathrm{HH}}=1.9 \mathrm{~Hz}, 1 \mathrm{H}\right), 2.86(\mathrm{dd}$, ${ }^{2} J_{\mathrm{HH}}=15.5 \mathrm{~Hz}$ and $\left.{ }^{3} J_{\mathrm{HH}}=6.8 \mathrm{~Hz}, 1 \mathrm{H}\right), 2.57-2.50(\mathrm{~m}, 1 \mathrm{H}), 2.37\left(\mathrm{~d},{ }^{2} J_{\mathrm{HH}}=15.9 \mathrm{~Hz}, 1 \mathrm{H}\right)$. ${ }^{13} \mathrm{C} \mathrm{NMR}\left(\mathrm{CDCl}_{3}\right): \delta 207.0,162.1\left(\mathrm{~d},{ }^{1} J_{\mathrm{CF}}=246.5 \mathrm{~Hz}\right), 155.3,136.1,135.5,128.6,128.5(\mathrm{~d}$, $\left.{ }^{3} J_{\mathrm{CF}}=5.6 \mathrm{~Hz}\right), 128.3,128.0,115.7\left(\mathrm{~d},{ }^{2} J_{\mathrm{CF}}=21.2 \mathrm{~Hz}\right), 67.8,54.1,44.2,40.5,38.9$.<smiles>Cc1cc(C)cc(C2CC(=O)CCN2C(=O)OCc2ccccc2)c1</smiles>

Table 2, entry $5((R)-2 e)$. The reaction was stirred for $7 \mathrm{~h}$ with 3.0 equiv of 3,5$\mathrm{Me}_{2} \mathrm{C}_{6} \mathrm{H}_{3} \mathrm{ZnCl}$ in the presence of $[\mathrm{RhCl}((R)-\text { binap })]_{2}(6 \mathrm{~mol} \% \mathrm{Rh})$. Yellow oil. $87 \%$ yield. The ee was determined on a Daicel Chiralcel OD-H column with hexane : isopropanol $=90: 10$, flow $=1.0 \mathrm{~mL} / \mathrm{min}$. Retention times: $16.1 \mathrm{~min}[(S)-$ enantiomer], $21.6 \mathrm{~min}\left[(R)\right.$-enantiomer]. $99 \%$ ee. $[\alpha]^{20}{ }_{\mathrm{D}}+74.7\left(c \mathrm{c} 1.13, \mathrm{CHCl}_{3}\right)$. The absolute configuration was assigned by analogy with equation 2 .

${ }^{1} \mathrm{H}$ NMR $\left(\mathrm{CDCl}_{3}\right): \delta$ 7.36-7.32 (m, 5H), $6.90(\mathrm{~s}, 1 \mathrm{H}), 6.82(\mathrm{~s}, 2 \mathrm{H}), 5.75(\mathrm{bs}, 1 \mathrm{H}), 5.26$ $\left(\mathrm{d},{ }^{2} J_{\mathrm{HH}}=12.3 \mathrm{~Hz}, 1 \mathrm{H}\right), 5.19\left(\mathrm{~d},{ }^{2} J_{\mathrm{HH}}=12.2 \mathrm{~Hz}, 1 \mathrm{H}\right), 4.27(\mathrm{bs}, 1 \mathrm{H}), 3.21\left(\mathrm{t},{ }^{3} J_{\mathrm{HH}}=10.9\right.$ $\mathrm{Hz}, 1 \mathrm{H}), 2.96\left(\mathrm{dd},{ }^{2} J_{\mathrm{HH}}=15.5 \mathrm{~Hz}\right.$ and $\left.{ }^{3} J_{\mathrm{HH}}=2.0 \mathrm{~Hz}, 1 \mathrm{H}\right), 2.82\left(\mathrm{dd},{ }^{2} J_{\mathrm{HH}}=15.5 \mathrm{~Hz}\right.$ and $\left.{ }^{3} J_{\mathrm{HH}}=7.0 \mathrm{~Hz}, 1 \mathrm{H}\right), 2.55-2.48(\mathrm{~m}, 1 \mathrm{H}), 2.37\left(\mathrm{~d},{ }^{2} J_{\mathrm{HH}}=16.0 \mathrm{~Hz}, 1 \mathrm{H}\right), 2.27(\mathrm{~s}, 6 \mathrm{H}) .{ }^{13} \mathrm{C}$ NMR $\left(\mathrm{CDCl}_{3}\right): \delta 207.3,155.4,139.6,138.3,136.3,129.3,128.5,128.1,127.9,124.4,67.6$, 54.4, 44.2, 40.5, 38.9, 21.3. HRMS (ESI) calcd for $\mathrm{C}_{21} \mathrm{H}_{23} \mathrm{NO}_{3} \mathrm{Na}\left(\mathrm{M}+\mathrm{Na}^{+}\right)$360.1570, found 360.1570 .

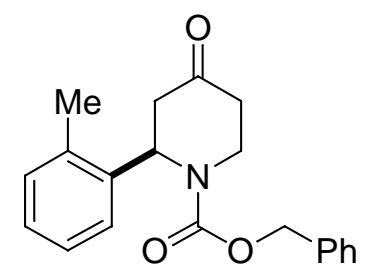

Table 2, entry $6((\boldsymbol{R})-2 \mathrm{f})$. The reaction was stirred for $4 \mathrm{~h}$ with 3.0 equiv of 2$\mathrm{MeC}_{6} \mathrm{H}_{4} \mathrm{ZnCl}$ in the presence of $[\mathrm{RhCl}((R) \text {-binap })]_{2}(6 \mathrm{~mol} \% \mathrm{Rh})$. Yellow oil. $100 \%$ yield. The ee was determined on a Daicel Chiralcel OD-H column with hexane : isopropanol $=95: 5$, flow $=1 \mathrm{~mL} / \mathrm{min}$. Retention times: $26.0 \mathrm{~min}$ [(R)-enantiomer], 29.3 min $\left[(S)\right.$-enantiomer]. $99 \%$ ee. $[\alpha]^{20}{ }_{\mathrm{D}}+75.9$ (c $\left.0.47, \mathrm{CHCl}_{3}\right)$. The absolute configuration was assigned by analogy equation 2 .

${ }^{1} \mathrm{H}$ NMR $\left(\mathrm{CDCl}_{3}\right): \delta 7.35-7.29(\mathrm{~m}, 3 \mathrm{H}), 7.23-7.18(\mathrm{~m}, 6 \mathrm{H}), 5.75(\mathrm{bs}, 1 \mathrm{H}), 5.18\left(\mathrm{~d},{ }^{2} J_{\mathrm{HH}}\right.$ $=12.0 \mathrm{~Hz}, 1 \mathrm{H}), 5.14\left(\mathrm{~d},{ }^{3} \mathrm{~J}_{\mathrm{HH}}=12.2 \mathrm{~Hz}, 1 \mathrm{H}\right), 4.28(\mathrm{bs}, 1 \mathrm{H}), 3.25(\mathrm{bs}, 1 \mathrm{H}), 2.86\left(\mathrm{dd},{ }^{2} J_{\mathrm{HH}}\right.$ $=15.6 \mathrm{~Hz}$ and $\left.{ }^{3} J_{\mathrm{HH}}=4.4 \mathrm{~Hz}, 1 \mathrm{H}\right), 2.80\left(\mathrm{dd},{ }^{2} \mathrm{~J}_{\mathrm{HH}}=15.4 \mathrm{~Hz}\right.$ and $\left.{ }^{3} J_{\mathrm{HH}}=6.6 \mathrm{~Hz}, 1 \mathrm{H}\right)$, 2.58-2.46 (m, 1H), $2.46\left(\mathrm{~d},{ }^{2} J_{\mathrm{HH}}=17.1 \mathrm{~Hz}, 1 \mathrm{H}\right), 2.26(\mathrm{~s}, 3 \mathrm{H}) .{ }^{13} \mathrm{C} \mathrm{NMR}\left(\mathrm{CDCl}_{3}\right): \delta 207.8$, 
155.1, 138.6, 136.4, 136.1, 131.1, 128.5, 128.1, 127.9, 127.8, 126.2, 126.1, 67.7, 52.8, 44.7, 40.8, 38.9, 19.2. Anal. Calcd for $\mathrm{C}_{20} \mathrm{H}_{21} \mathrm{NO}_{3}$ : C, 74.28; $\mathrm{H}, 6.55$. Found: $\mathrm{C}, 74.09 ; \mathrm{H}, 6.56$.

\section{Procedure for Equation 2.}<smiles>Cc1cc(F)ccc1C1CC(=O)CCN1C(=O)OCc1ccccc1</smiles>

4-Fluoro-2-methylphenylzinc chloride $(1.54 \mathrm{~mL}, 0.80 \mathrm{mmol} ; 0.52 \mathrm{M}$ solution in THF) was added dropwisely to a stirring solution of $1(46.3 \mathrm{mg}, 0.20 \mathrm{mmol})$ and $[\mathrm{RhCl}((R) \text {-binap })]_{2}(12.2 \mathrm{mg}, 16.0 \mu \mathrm{mol} \mathrm{Rh})$ in THF $(0.5 \mathrm{~mL})$, and the mixture was stirred for $9 \mathrm{~h}$ at $20^{\circ} \mathrm{C}$. After quenching with $\mathrm{H}_{2} \mathrm{O}(60 \mu \mathrm{L})$, the reaction mixture was passed through a pad of silica gel with $\mathrm{Et}_{2} \mathrm{O}$, and the solvent was then removed under vacuum. The residue was chromatographed on silica gel with $\mathrm{Et}_{2} \mathrm{O} /$ hexane = $1 / 1$ to afford the desired 1,4-adduct (CAS 414910-00-2) as a pale yellow oil (55.7 mg, $0.163 \mathrm{mmol} ; 82 \%$ yield).

The ee was determined on a Daicel Chiralcel OD-H column with hexane : isopropanol $=90: 10$, flow $=1.0 \mathrm{~mL} / \mathrm{min}$. Retention times: $20.5 \mathrm{~min}[(R)$ enantiomer], $23.4 \mathrm{~min}\left[(S)\right.$-enantiomer]. 97\% ee. $[\alpha]_{\mathrm{D}}^{20}+59.5\left(c 0.61, \mathrm{CHCl}_{3}\right)$.

${ }^{1} \mathrm{H}$ NMR $\left(\mathrm{CDCl}_{3}\right): \delta$ 7.38-7.23 $(\mathrm{m}, 5 \mathrm{H}), 7.13-7.10(\mathrm{~m}, 1 \mathrm{H}), 6.87-6.82(\mathrm{~m}, 2 \mathrm{H}), 5.71(\mathrm{bs}$, $1 \mathrm{H}), 5.19\left(\mathrm{~d},{ }^{2} J_{\mathrm{HH}}=12.1 \mathrm{~Hz}, 1 \mathrm{H}\right), 5.14\left(\mathrm{~d},{ }^{2} J_{\mathrm{HH}}=12.3 \mathrm{~Hz}, 1 \mathrm{H}\right), 4.28(\mathrm{bs}, 1 \mathrm{H}), 3.21(\mathrm{bs}$, $1 \mathrm{H}), 2.84-2.76(\mathrm{~m}, 2 \mathrm{H}), 2.57-2.50(\mathrm{~m}, 1 \mathrm{H}), 2.46\left(\mathrm{~d},{ }^{2} \mathrm{~J}_{\mathrm{HH}}=16.6 \mathrm{~Hz}, 1 \mathrm{H}\right), 2.25(\mathrm{~s}, 3 \mathrm{H}) .{ }^{13} \mathrm{C}$ $\operatorname{NMR}\left(\mathrm{CDCl}_{3}\right): \delta 207.6,162.0\left(\mathrm{~d},{ }^{1} J_{\mathrm{CF}}=247.0 \mathrm{~Hz}\right), 155.1,139.0,136.0,134.3,128.5,128.2$, 128.0, 127.7, $117.9\left(\mathrm{~d},{ }^{2} J_{\mathrm{CF}}=21.7 \mathrm{~Hz}\right), 112.7\left(\mathrm{~d},{ }^{2} J_{\mathrm{CF}}=21.2 \mathrm{~Hz}\right), 67.8,52.3,44.8,40.8$, $38.9,19.2$.

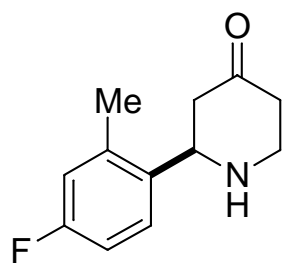

The 1,4-adduct obtained above (32.9 mg, $96.4 \mu \mathrm{mol})$ was dissolved in $\mathrm{MeOH}(0.5$ $\mathrm{mL})$ and $\mathrm{Pd} / \mathrm{C}(10 \% ; 8.8 \mathrm{mg}, 8.3 \mu \mathrm{mol} \mathrm{Pd})$ was added to it. $\mathrm{H}_{2}$ gas ( 1 atm) was then purged and the mixture was stirred for $4 \mathrm{~h}$ at $20^{\circ} \mathrm{C}$. After releasing $\mathrm{H}_{2}, \mathrm{Pd} / \mathrm{C}$ was removed by filtration through celite with $\mathrm{Et}_{2} \mathrm{O}$. The resulting solution was washed with aqueous $\mathrm{NaOH}$, dried over $\mathrm{MgSO}_{4}$, filtered, and then concentrated under vacuum to afford compound (R)-3 (CAS 414910-12-6 for $(R)$-enantiomer; 414909-99-2 for racemate) as an yellow oil $(17.7 \mathrm{mg}, 85.4 \mu \mathrm{mol} ; 89 \%$ yield $) .[\alpha]_{D}^{20}+77.4(c 0.18$, DMSO). The absolute configuration was determined by comparison of the optical rotation with the literature value. ${ }^{7}$

${ }^{1} \mathrm{H} \mathrm{NMR}\left(\mathrm{CDCl}_{3}\right): \delta 7.57\left(\mathrm{dd},{ }^{3} J_{\mathrm{HH}}=8.4 \mathrm{~Hz}\right.$ and $\left.{ }^{4} J_{\mathrm{HF}}=5.8 \mathrm{~Hz}, 1 \mathrm{H}\right), 6.94-6.90(\mathrm{~m}$, $1 \mathrm{H}), 6.88\left(\mathrm{dd},{ }^{3} J_{\mathrm{HF}}=9.6 \mathrm{~Hz}\right.$ and $\left.{ }^{4} J_{\mathrm{HH}}=2.4 \mathrm{~Hz}, 1 \mathrm{H}\right), 4.17\left(\mathrm{dd},{ }^{3} J_{\mathrm{HH}}=11.5 \mathrm{~Hz}\right.$ and ${ }^{3} J_{\mathrm{HH}}=$

\footnotetext{
${ }^{7}$ Alvaro, G.; Di Fabio, R.; Tranquillini, M. E.; Spada, S. Application: WO 2003-EP1308 20030210.
} 
3.2 Hz, 1H), 3.50-3.46 (m, 1H), $3.05\left(\mathrm{td}, J_{\mathrm{HH}}=12.3 \mathrm{~Hz}\right.$ and $\left.{ }^{3} J_{\mathrm{HH}}=3.2 \mathrm{~Hz}, 1 \mathrm{H}\right), 2.71(\mathrm{td}$, $J_{\mathrm{HH}}=13.6 \mathrm{~Hz}$ and $\left.{ }^{3} J_{\mathrm{HH}}=6.7 \mathrm{~Hz}, 1 \mathrm{H}\right), 2.61-2.45(\mathrm{~m}, 3 \mathrm{H}), 2.35(\mathrm{~s}, 3 \mathrm{H})$.

\section{Procedure for Equation 3.}

$\mathrm{PhZnCl}(0.55 \mathrm{~mL}, 0.30 \mathrm{mmol} ; 0.55 \mathrm{M}$ solution in THF) was added dropwisely to a stirring solution of $1(0.20 \mathrm{mmol})$ and $[\mathrm{RhCl}((R)-\text { binap })]_{2}(4.6 \mathrm{mg}, 6.0 \mu \mathrm{mol} \mathrm{Rh})$ in THF $(0.5 \mathrm{~mL})$, and the mixture was stirred for $2 \mathrm{~h}$ at $20^{\circ} \mathrm{C}$. After cooling to $0{ }^{\circ} \mathrm{C}$, an electrophile $(0.4 \mathrm{mmol})$ was added dropwisely and the resulting solution was further stirred for $2 \mathrm{~h}$ at $20^{\circ} \mathrm{C}$. The mixture was then passed through a pad of silica gel with $\mathrm{Et}_{2} \mathrm{O}$ and the solvent was removed under vacuum. The residue was purified by silica gel PTLC with $\mathrm{Et}_{2} \mathrm{O} /$ hexane $=1 / 1$ to afford the desired product.<smiles>C=CC[C@H]1C(=O)CCN(C(=O)Cc2ccccc2)C1c1ccccc1</smiles>

Compound 5. Pale yellow oil. $83 \%$ yield. The ee was assumed to be $>99.5 \%$ $(2 R, 3 S)$ based on the result in Table 1 , entry 4 . $[\alpha]^{20}{ }_{\mathrm{D}}+119.8$ (c 1.01, $\left.\mathrm{CHCl}_{3}\right)$. The absolute configuration was assigned by analogy with equation 2 .

${ }^{1} \mathrm{H}$ NMR $\left(\mathrm{CDCl}_{3}\right): \delta$ 7.39-7.23 (m, 10H), 5.78-5.68 (m, 1H), $5.58(\mathrm{bs}, 1 \mathrm{H}), 5.24(\mathrm{~d}$, $\left.{ }^{2} J_{\mathrm{HH}}=12.2 \mathrm{~Hz}, 1 \mathrm{H}\right), 5.21\left(\mathrm{~d},{ }^{2} J_{\mathrm{HH}}=12.3 \mathrm{~Hz}, 1 \mathrm{H}\right), 5.10-5.02(\mathrm{~m}, 2 \mathrm{H}), 4.32(\mathrm{bs}, 1 \mathrm{H}), 3.18$ $\left(\mathrm{ddd},{ }^{2} J_{\mathrm{HH}}=13.8 \mathrm{~Hz}\right.$ and ${ }^{3} \mathrm{~J}_{\mathrm{HH}}=11.8 \mathrm{~Hz}$ and $\left.4.1 \mathrm{~Hz}, 1 \mathrm{H}\right), 3.05\left(\mathrm{td},{ }^{3} J_{\mathrm{HH}}=7.0 \mathrm{~Hz}\right.$ and $2.6 \mathrm{~Hz}, 1 \mathrm{H}), 2.65-2.56(\mathrm{~m}, 1 \mathrm{H}), 2.46-2.34(\mathrm{~m}, 2 \mathrm{H}), 2.32\left(\mathrm{~d},{ }^{2} \mathrm{~J}_{\mathrm{HH}}=16.0 \mathrm{~Hz}, 1 \mathrm{H}\right) .{ }^{13} \mathrm{C}$ $\operatorname{NMR}\left(\mathrm{CDCl}_{3}\right): \delta 209.4,155.8,139.4,136.2,134.1,128.7,128.5,128.2,128.0,127.8,127.1$, $118.2,67.8,58.1,52.8,38.9,38.5,34.9$. Anal. Calcd for $\mathrm{C}_{22} \mathrm{H}_{23} \mathrm{NO}_{3}: \mathrm{C}, 75.62 ; \mathrm{H}, 6.63$. Found: C, 75.44; H, 6.59.<smiles>CC(C)(C)C(=O)OC1=CC(c2ccccc2)N(C(=O)OCc2ccccc2)CC1</smiles>

Compound 6. Colorless oil. 97\% yield. The ee was assumed to be $>99.5 \%(R)$ based on the result in Table 1 , entry $4 .[\alpha]^{20}+114.8\left(c 0.96, \mathrm{CHCl}_{3}\right)$. The absolute configuration was assigned by analogy with equation 2 .

${ }^{1} \mathrm{H} \mathrm{NMR}\left(\mathrm{CDCl}_{3}, 45^{\circ} \mathrm{C}\right): \delta 7.44$ (bs, $\left.1 \mathrm{H}\right), 7.37-7.28(\mathrm{~m}, 9 \mathrm{H}), 5.85$ (bs $\left.1 \mathrm{H}\right), 5.55$ (bs, $1 \mathrm{H}), 5.22\left(\mathrm{~d},{ }^{2} J_{\mathrm{HH}}=12.3 \mathrm{~Hz}, 1 \mathrm{H}\right), 5.15\left(\mathrm{~d},{ }^{2} J_{\mathrm{HH}}=12.3 \mathrm{~Hz}, 1 \mathrm{H}\right), 4.21(\mathrm{bs}, 1 \mathrm{H}), 3.12(\mathrm{ddd}$, ${ }^{2} J_{\mathrm{HH}}=13.6 \mathrm{~Hz}$ and ${ }^{3} \mathrm{~J}_{\mathrm{HH}}=12.0 \mathrm{~Hz}$ and $\left.4.1 \mathrm{~Hz}, 1 \mathrm{H}\right), 2.61-2.52(\mathrm{~m}, 1 \mathrm{H}), 2.08\left(\mathrm{~d},{ }^{2} \mathrm{~J}_{\mathrm{HH}}=\right.$ $13.3 \mathrm{~Hz}, 1 \mathrm{H}), 1.28(\mathrm{~s}, 9 \mathrm{H}) .{ }^{13} \mathrm{C} \mathrm{NMR}\left(\mathrm{CDCl}_{3}\right): \delta 176.9,154.9,147.8,140.0,136.5,128.51$, $128.48,128.46,128.03,127.97,127.8,113.7,67.5,67.4,54.0,38.9,36.9,27.1$. Anal. Calcd for $\mathrm{C}_{24} \mathrm{H}_{27} \mathrm{NO}_{4}$ : C, 73.26; $\mathrm{H}$, 6.92. Found: $\mathrm{C}, 73.37 ; \mathrm{H}, 7.19$.

\section{Procedure for Equations 4 and 5.}


$\mathrm{PhZnCl}(0.55 \mathrm{~mL}, 0.30 \mathrm{mmol} ; 0.55 \mathrm{M}$ solution in THF) was added dropwisely to a stirring solution of $\alpha, \beta$-enone $(0.20 \mathrm{mmol})$ and $[\mathrm{RhCl}((R) \text {-binap })]_{2}(4.6 \mathrm{mg}, 6.0 \mu \mathrm{mol}$ $\mathrm{Rh})$ in THF $(0.5 \mathrm{~mL})$, and the mixture was stirred for $1 \mathrm{~h}$ at $20^{\circ} \mathrm{C}$. After quenching with $\mathrm{H}_{2} \mathrm{O}(0.1 \mathrm{~mL})$, the reaction mixture was passed through a pad of silica gel with $\mathrm{Et}_{2} \mathrm{O}$, and the solvent was then removed under vacuum. The residue was purified by silica gel PTLC with EtOAc/hexane $=1 / 3$ to afford the desired 1,4-adduct.

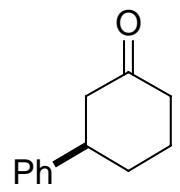

Equation 4 ((R)-7). (CAS 34993-51-6) Colorless oil. 98\% yield. The ee was determined on a Daicel Chiralcel OD-H column with hexane : isopropanol $=98: 2$, flow $=0.5 \mathrm{~mL} / \mathrm{min}$. Retention times: $24.0 \mathrm{~min}[(S)$-enantiomer], $25.6 \mathrm{~min}[(R)-$ enantiomer]. $99 \%$ ee.<smiles>C[PH+]C([PH])CC(C)=O</smiles>

Equation 5 ((R)-8). (CAS 162239-77-2) Colorless oil. 99\% yield. The ee was determined on a Daicel Chiralcel OD-H column with hexane : isopropanol $=98: 2$, flow $=0.5 \mathrm{~mL} / \mathrm{min}$. Retention times: $19.2 \mathrm{~min}[(R)$-enantiomer], $21.9 \mathrm{~min}[(S)$ enantiomer]. $94 \%$ ee. 